\title{
Efectos socioeconómicos del programa de las casas de acogida en Andalucía (España)
}

\section{Socio-economic effects of the programme of women's shelters in Andalusia (Spain)}

doi: http://dx.doi.org/10.32870/

espiral.v25i71.6386

\section{Resumen}

Este trabajo, partiendo de datos oficiales sobre mujeres ingresadas en casas de acogida andaluzas, aplica técnicas estadísticas para diferenciar el perfil de aquellas mujeres que regresan con el agresor del perfil de las que optan por cualquier otra residencia, ambas tras someterse al plan de recuperación integral (psicológico, jurídico y socioeducativo). Entre las conclusiones, se obtiene que aspectos como la nacionalidad, la capacidad económica o el número de hijos son factores determinantes en la decisión de residencia tras el plan de recuperación integral.

Palabras clave: violencia de género, ley integral, casas de acogida, análisis estadístico de datos, Andalucía.
Juana de los Ángeles Toledo-Larrea

María Isabel Sánchez-Rodríguez*•

\begin{abstract}
This work, considering official data of users of women's shelter in Andalusia, applies statistical techniques to differentiate between the profile of the women that return with the abuser and the profile of the women that choose any other residence, both after undergoing the plan of integral recovery (psychological, juridical and socio-educational).The conclusions highlight that aspects like the nationality, the economic capacity or the number of children are determinant factors on residence decision before the integral recovery plan.
\end{abstract}

Keywords: Gender-based violence, integrated law, women's shelters, statistical data analysis, Andalusia.

\footnotetext{
-Asesora legal de los Recursos Integrales de Protección y Acogida de la Junta de Andalucía, España. ORCID: http://orcid.org/0000-0002-005I-850X.

toledolarrea.nani@andaluciajunta.es

- Profesora-Investigadora del Departamento de Estadística y Empresa, Universidad de Córdoba, España. ORCID: http://orcid.org/0000-0003-0657-7823.

tdlsarom@uco.es

Fecha de recepción: 14 de diciembre de 2016. Fecha de aceptación: 09 de agosto de 2017.
} 


\section{Introducción}

La Convención Interamericana para Prevenir, Sancionar y Erradicar la Violencia contra la Mujer (Belém do Pará) de 1994 establece que la violencia de género constituye una "violación de los derechos humanos y las libertades fundamentales y limita total o parcialmente a la mujer el reconocimiento, goce y ejercicio de tales derechos y libertades" (Convención Interamericana para Prevenir, Sancionar y Erradicar la Violencia contra la Mujer, 1994, p. 1). La Organización de las Naciones Unidas reconoció, en la IV Conferencia Mundial sobre la Mujer, celebrada en Beijing en 1995, que la violencia de género impide el logro de los objetivos de igualdad, desarrollo y paz, puesto que viola y menoscaba o impide a las mujeres el disfrute de los derechos humanos y las libertades fundamentales (IV Conferencia Mundial de la Mujer, 1995, p. 51).

Son muchas las definiciones existentes que intentan explicar conceptualmente el fenómeno de la violencia de género contra las mujeres. Así, por ejemplo, Lorente, Lorente y Martínez Vilda (2000) denominan síndrome de agresión a la mujer al conjunto de

agresiones sufridas por la mujer como consecuencia de los condicionantes socioculturales que actúan sobre el género masculino y femenino, situándola en una posición de subordinación al hombre, y manifestadas en los tres ámbitos básicos de relación de la persona: maltrato en el medio familiar, agresión sexual en la vida en sociedad y acoso en el medio laboral (p. 3).

Además, estos autores denominan sindrome de maltrato a la mujer al "conjunto de lesiones físicas y psíquicas resultantes de las agresiones repetidas llevadas a cabo por el hombre sobre su cónyuge o mujer a la que estuviese o haya estado unido por análogas relaciones de afectividad" (p. 4). 
Hasta el año 2004, el sistema que existía en España, similar al de algunos países europeos, ${ }^{1}$ consistía en modificar distintos ámbitos legislativos, al tiempo que se adoptaban medidas de carácter social y se creaban organismos nacionales para intentar conocer a fondo el problema de la violencia de género. El 28 de diciembre de 2004, se redactó la Ley Orgánica 1/2004, de Medidas de Protección Integral contra la Violencia de Género, mejor conocida como Ley Integral. Esta constituye la primera normativa que enfrenta el problema de la violencia de género de manera global. Así, usando un procedimiento similar al empleado por algunos sistemas latinoamericanos, ${ }^{2}$ se recogen en un mismo texto legal medidas de carácter administrativo, educativo y social y modificaciones al Código Penal, al Civil y a las leyes procesales, continuando un proceso de reformas legales en todos los ámbitos para promover la igualdad de las mujeres y buscando, a través del derecho penal y de la prevención general, solucionar este problema social de primera magnitud.

La Ley Integral declara en su exposición de motivos que la violencia de género se manifiesta como un símbolo

I.Tres ejemplos. El Código Civil de Alemania regula el delito de malos tratos en su artículo 225, modificado por una ley del 01 de abril de 1998; el delito de malos tratos se concibe para proteger a todas las personas que están en una situación de dependencia o subordinación con respecto a otro. En el sistema portugués, el código penal regula el delito de malos tratos en su artículo I52, que señala como infractor a quien, por motivos crueles, daña corporalmente a niños, incapaces y cónyuges. En el sistema francés, el Código Penal castiga el delito de lesiones dependiendo del resultado causado, pero el delito se agrava si el sujeto pasivo es menor de 15 años, si la persona es especialmente vulnerable en función de la edad, enfermedad o deficiencia, y si el delito es cometido sobre ascendientes o sobre el cónyuge, entendiéndose el delito de violencia familiar como un delito cualificado de lesiones. 2. Los países latinoamericanos abordan este problema mediante la elaboración de una ley especial que recoge en el mismo cuerpo legal los aspectos penales, sociales, civiles, procesales, etc. Estas leyes específicas son, entre otras: la Ley 7-12-94, de Argentina, sobre protección de la violencia familiar; la Ley 15-12-95, de Bolivia, contra la violencia familiar; la Ley 27-8-9, de Chile, sobre violencia intrafamiliar; y la Ley 16-96, de Colombia, sobre normas para prevenir, remediar y sancionar la violencia familiar. 
de la desigualdad existente en nuestra sociedad, que los poderes públicos no pueden ser ajenos a este problema que constituye uno de los ataques más flagrantes a los derechos fundamentales, y que la ley pretende seguir las recomendaciones de los organismos internacionales, en el sentido de proporcionar una respuesta global a la violencia que se ejerce sobre las mujeres, por lo que su texto abarca aspectos preventivos, educativos, sociales y de atención posterior a las víctimas.

Las medidas que adopta la Ley Integral son estrictamente necesarias en todos los ámbitos. Incluyen, por ejemplo, las medidas educativas necesarias para transmitir al conjunto de la sociedad nuevas escalas de valores basadas en el respeto de los derechos humanos, las libertades fundamentales y la igualdad entre hombres y mujeres, y, de igual modo, las medidas de intervención inmediata que protejan la integridad de las mujeres maltratadas y contribuyan a su integridad laboral y social, alejada del maltratador.

Debido a la importancia que esta ley supuso en el panorama legislativo español, la violencia de género ha sido objeto de numerosos estudios en los últimos años, que abarcan los distintos ámbitos contemplados en dicha ley: el educativo, el de la publicidad y los medios de comunicación, el sanitario, el social, el jurídico-penal y el económico.

Así, trabajos como los de Ferrer, Bosch y Navarro (2011), Montilla, Pérez y Montes (2010), Segura (2012) y Zayas (2006) analizan la violencia de género desde el ámbito de la educación, mientras los de Capella, et al. (2010), Martín Llaguno y Navarro Beltrá (2013), Segura (2012) y Yoon y Kim (2014) tratan aspectos relacionados con la publicidad y los medios de comunicación.

Los profesionales sanitarios juegan un papel fundamental en la prevención, detección y atención integral a las mujeres afectadas por violencia de género, por lo que trabajos como los de Cabrera y Granero (2011), Coll Vinent, et al. 
(2007; 2008), Djikanovic, King y Bjegovic (2013), Muslera, et al. (2009), Rangel da Silva, et al. (2011) y Vives Cases, et al. (2007) analizan la violencia de género desde el ámbito de la sanidad. Trabajos como los de Benagiano, Carrara y Fillipi (2010), Ferrer Pérez y Bosch Fiol (2014), Hearn y McKie (2010), Mulla y Hlavka (2011), Puigvert (2014) y Zuckerhut (2010) contemplan la violencia de género desde la perspectiva de la asistencia social. Finalmente, artículos como los de Argueelles, et al. (2009), Azevedo (2008), Corcoy (2010), Lemaitre y Sandvik (2014), Martín Martínez (2011) y Molina, Martínez Ortega y Guancha (2013) consideran la violencia de género en el ámbito penal y judicial.

La Ley Integral también supuso la regulación de los recursos de atención y acogida para mujeres víctimas de violencia de género, cuyas competencias son transferidas a las comunidades autónomas (artículo 19). En particular, en Andalucía, la Ley 13/2007, del 26 de noviembre, distingue los siguientes niveles de actuación para mujeres víctimas de violencia de género y los menores que las acompañan (artículo 44): 1) los centros de emergencia, que ofrecen a las mujeres una acogida inmediata y temporal, de corta duración, mientras se valora el recurso social más adecuado a sus circunstancias personales; 2) las casas de acogida, que garantizan una acogida temporal y una atención integral multidisciplinar para que las mujeres sean capaces de recuperarse de los efectos de la violencia padecida; y 3) los pisos tutelados, que son viviendas cedidas para uso familiar, con carácter temporal, a aquellas mujeres que pueden vivir de forma independiente.

En particular, este trabajo enfoca su atención en las casas de acogida andaluzas, abordando un estudio estadístico sobre las características de las mujeres usuarias de las mismas durante los años 2004-2010, años en los que, como se ha indicado anteriormente, se produjeron importantes 
cambios legislativos a nivel estatal y autonómico en materia de violencia de género.

Por otra parte, el reciente Acuerdo de la Conferencia Sectorial de Igualdad, del 21 de julio de 2014 (resolución del 09 de junio de 2015), contempla que las necesidades de protección y seguridad de la mujer víctima de violencia de género conllevan en ocasiones la conveniencia de alejarse de su agresor y comenzar un proceso de recuperación y empoderamiento de tipo multidisciplinar fuera del ámbito de la comunidad autónoma de residencia. Por este motivo, este trabajo pretende contrastar los perfiles socioeconómicos de las usuarias de las casas de acogida andaluzas que regresan al domicilio con el agresor con los de aquellas que optan por otro tipo de residencia.

La literatura recoge muchos trabajos en los que se realiza un tratamiento estadístico de datos relacionados con violencia de género. Las técnicas estadísticas específicas que se han utilizado en los últimos años en dichos trabajos son diversas. Así, por ejemplo, Echauri, et al. (2013) hacen uso de estadística descriptiva, el test chi-cuadrado y modelos ANOVA para estudiar la efectividad de un programa de tratamiento con inmigrantes que ejercen violencia de género contra la pareja. Los modelos de regresión logística son ampliamente usados, por ejemplo, por Djikanovic, King y Bjegovic (2013), para detectar diferencias de género en denuncias sobre violencia familiar en Serbia y su asociación con síntomas de salud, y por Epstein, et al. (2013) y Ruan, et al. (2010), para analizar los efectos del alcohol en la violencia. Fernández-Montalvo, et al. (2011) utilizan estadística descriptiva, contrastes de comparación de medias y tests chi-cuadrado para determinar el perfil diferencial de hombres maltratadores nacionales e inmigrantes. Ferrer Pérez y Bosch Fiol (2014) utilizan procedimientos gráficos y tests chi-cuadrado en el análisis de la violencia de género como un problema social en España. Martín Llaguno y Navarro 
Beltrá (2013) usan tablas de frecuencias y pruebas chi-cuadrado para valorar, a través de anuncios publicitarios seleccionados de Argentina, España, Estados Unidos y México, el impacto de las normas sobre violencia de género. Vives Cases, et al. (2007) utilizan gráficos, medidas descriptivas, tasas e intervalos de confianza para explorar la distribución geográfica de la mortalidad y las denuncias por violencia del compañero íntimo. Da Mota, Godoi y De Assis (2007; 2008) y Rodríguez Menés, Puig y Sobrino (2014) utilizan análisis de correspondencias múltiple para determinar el perfil de mujeres víctimas de violencia de género en Brasil y para diferenciar entre mujeres españolas víctimas de violencia en varios o un solo ámbito de su vida, respectivamente.

Este trabajo hace uso de técnicas estadísticas para caracterizar socioeconómicamente a las mujeres usuarias de casas de acogida andaluzas, con especial interés en diferenciar el perfil de aquellas que, después de haber permanecido ingresadas en una casa de acogida y haber sido sometidas al proceso de recuperación integral (socioeducativa, social, formativa, psicológica y jurídica) que esta estancia conlleva, deciden volver al domicilio conyugal con el agresor.

Contrariamente a algunos trabajos citados, se hace uso de una fuente de datos oficial de la Junta de Andalucía, que proporciona información sobre un número muy elevado de mujeres (1 953). La disponibilidad de un conjunto de datos de esta magnitud es una característica positiva de todo estudio estadístico en tanto aumenta la fiabilidad del mismo y la validez de sus conclusiones. Además, estos datos se refieren a mujeres ingresadas en casas de acogida, aspecto que proporciona más valor al estudio, puesto que datos oficiales sobre casas de acogida de mujeres víctimas de violencia de género no han sido contemplados previamente en la literatura existente. Además, este trabajo hace uso de varias técnicas de análisis estadístico con la finalidad de analizar el grado de asociación entre ellas, determinar la estructura 
de interrelaciones que subyace entre sus modalidades o estudiar las diferencias que existen para una variable respecto a los grupos de casos que establece otra.

\section{Las casas de acogida andaluzas}

La Comunidad Autónoma de Andalucía asume en su Estatuto de Autonomía (artículo 16) (Boletín Oficial de la Junta de Andalucía, 2007a) un fuerte compromiso con la erradicación de la violencia de género y la protección integral a las mujeres. La Ley 10/1988, en su artículo 30 (Boletín Oficial de la Junta de Andalucía, 1988), creó el Instituto Andaluz de la Mujer como organismo responsable de "promover las condiciones para que sea real y efectiva la igualdad del hombre y la mujer andaluces, haciendo posible la participación y presencia de la mujer en la vida política, económica, cultural y social, y superando cualquier discriminación laboral, cultural, económica o política de la mujer" (p. 5532). La Ley 13/2007, del 26 de noviembre, responde al desarrollo específico de las estrategias contra la violencia de género y constituye el reconocimiento de los derechos de las mujeres en orden de su protección y atención.

El artículo 43 de la Ley 13/2007 establece que "la Administración de la Junta de Andalucía garantizará a las mujeres víctimas de violencia de género la acogida, la atención integral especializada y multidisciplinar, y los medios de apoyo y recuperación" (p. 25). Además, indica que "la atención integral, especializada y multidisciplinar comprenderá una intervención con las mujeres y menores a su cargo" (p. 25), basada en un sistema coordinado de servicios, recursos y de ayudas económicas y sociolaborales con las siguientes características: 1) especializados; 2) multidisciplinares, lo cual implica información, asesoramiento y seguimiento jurídico, apoyo social, atención psicológica, apoyo a la inserción laboral, atención a los hijos y menores que estén bajo 
su guarda y custodia, y seguimiento de las reclamaciones de los derechos de las mujeres; y 3) accesibles, que supone la adaptación de las estructuras y los servicios que se proporcionen a las mujeres con discapacidad y a las mujeres inmigrantes. Las medidas de recuperación integral están recogidas en el título III de la Ley 13/2007.

El artículo 44 de la misma ley establece los requisitos y la tipología de los centros de atención integral y acogida (la tipología los divide en recursos de emergencia, casas de acogida y pisos tutelados). En particular, el presente artículo contempla datos sobre casas de acogida, que, según el artículo 44, "ofrecen una acogida temporal a las mujeres y menores que las acompañen, garantizándoles una atención integral multidisciplinar, para que las mujeres sean capaces de recuperarse de los efectos de la violencia padecida" (p. 26). La Junta de Andalucía determina reglamentariamente los requisitos que deben reunir estos centros, que deben garantizar una cobertura de acuerdo a la demanda existente.

Así, el programa de casas de acogida en Andalucía persigue mejorar la autoestima y las habilidades sociales y laborales de las mujeres, su adquisición de conocimientos y su aprendizaje de hábitos normalizados. En las casas, se ofrecen modelos de convivencia no violentos y se potencian los intercambios con otras mujeres y la adquisición de nuevos modelos de conducta, creándose grupos de ayuda mutua. Asimismo, se fomentan la integración al mercado laboral y el acceso a recursos sociales, económicos, educativos y formativos.

El ingreso a casas de acogida se realiza después de haber valorado la situación de cada mujer y de que esta haya solicitado su ingreso en las mismas. En ocasiones, es la mujer víctima la que decide buscar otra alternativa, como la de establecerse con familiares o amigos. En otros casos, la mujer solicita el ingreso en una casa de acogida ante el 
juez en el momento en que este le concede la orden de protección y dicta una medida de alejamiento a su favor y la salida del domicilio del agresor. Si la mujer decide ingresar en el recurso, se analizan numerosas variantes, como la aceptación por parte de la misma de dicho ingreso, el apoyo familiar o la independencia económica.

Siempre como recurso alternativo a situaciones en las que las medidas legales no garantizan la seguridad de las víctimas, se crearon los dispositivos de alojamiento, es decir, como recursos de carácter temporal que dan una respuesta inmediata a la cobertura de necesidades básicas de las mujeres víctimas, así como de las de sus hijos menores. Dichos dispositivos constituyen una alternativa especialmente significativa en los casos de carencia de recursos económicos y la falta de apoyos sociales o familiares. Estos recursos potencian la recuperación de las mujeres que, en ocasiones, carecen de habilidades sociales, lo que agudiza su indefensión y vulnerabilidad, por lo que el alojamiento y las intervenciones que desde los mismos se pueden prestar constituyen aspectos importantes en su proceso de recuperación.

A pesar de las medidas adoptadas, siguen existiendo perfiles de mujeres que precisan de intervención especializada, añadida a la situación de violencia, por lo que no pueden ser atendidas desde los lugares de alojamiento. Se trata de mujeres con necesidades especiales por drogodependencia activa, problemas de salud mental o ejercicio de la prostitución. El artículo 45 de la Ley 13/2007 contempla la atención a colectivos especialmente vulnerables.

También es importante señalar que el 50\% de las mujeres que ingresan en casas de acogida, además de los problemas derivados de haber sufrido situaciones de violencia de género, suele presentar otros problemas adicionales: desestructuración familiar desde su familia de origen, carencia de redes de apoyo efectivas, precariedad económica, dependen- 
cia de los servicios sociales y escasa o nula experiencia laboral. Además, las mujeres acogidas se alejan de su entorno más próximo al ingresar en el centro, enfrentando su dolor alejadas del medio que conocen y sin el apoyo afectivo de familiares y amigos. No obstante, podemos afirmar que en las casas de acogida se refleja la actuación de la sociedad hacia las víctimas, ya que es una institución creada para ellas y sus hijos exclusivamente.

\section{Estudio estadístico}

En esta sección, se realiza un análisis estadístico de datos oficiales de mujeres ingresadas en casas de acogida andaluzas durante el periodo 2004-2010, en el que se han producido en España, en general, y en la comunidad autónoma de Andalucía, en particular, importantes cambios legislativos en materia de violencia de género, tal y como se comentó en la introducción del presente artículo.

El estudio pretende, especialmente, delimitar el perfil socioeconómico de las mujeres víctimas de violencia de género que, tras someterse al plan de recuperación integral (psicológico, jurídico y socioeducativo) de las casas de acogida, deciden regresar con su agresor a la salida del recurso, contra el perfil de aquellas que optan por cualquier otro domicilio a la salida.

\section{I. Datos y variables. Análisis estadístico descriptivo}

La red de centros de acogida en Andalucía cuenta con unos treinta y cuatro centros entre recursos de emergencia (nueve), pisos tutelados (diecisiete) y casas de acogida (ocho, compuestas por un total de cincuenta y dos pisos). En total, dispone de unas quinientas plazas. Sin embargo, en este trabajo son objeto de estudio solamente las mujeres víctimas de violencia de género ingresadas en casas de acogida andaluzas entre los años 2004-2010, es decir, no se 
han contemplado en el estudio las mujeres que ingresaron en recursos de emergencia o pisos tutelados.

Los datos proceden de las fichas que se completan al realizar el contrato de estancia en dichas casas de acogida. Se trata de un patrón estadillo que recoge todas las incidencias más significativas de las usuarias. Estas fichas son elaboradas por la empresa Andaluza de Gestión Integral de Servicios Especializados, S. L. (AGISE), encargada de gestionar los recursos integrales de atención y acogida para mujeres víctimas de violencia de género dependientes de la Junta de Andalucía, y están informatizadas en el Sistema de Información del Instituto Andaluz de la Mujer (SIAM).

Más concretamente, la matriz de datos objeto de estudio está formada por 1953 filas, que contienen información sobre las mujeres víctimas de violencia de género observadas, y diez columnas, que recogen la información sobre las siguientes variables estadísticas:

- Provincia: variable categórica o no numérica nominal que recoge la provincia andaluza de ingreso en el recurso (Almería, Cádiz, Córdoba, Huelva, Jaén, Málaga y Sevilla). Cádiz (15.7\%) y Sevilla (15.4\%) son las provincias con un mayor porcentaje de mujeres acogidas.

- Año: variable numérica que contempla los años de 2004 a 2010. El menor porcentaje de acogidas se registró en 2004 (12.7\%), al comienzo del estudio, mientras que el mayor número de ingresos corresponde al año 2007 (16.2\%).

- Edad: variable numérica que recoge la edad (en años) de la mujer en el momento del ingreso. La edad media y mediana es de 31.85 y 31 años, respectivamente. La edad mínima observada es de 18 años (mayoría de edad) y la máxima de 78 años. Sin embargo, la edad de 39 años acumula el $75 \%$ de los casos analizados (es el tercer cuartil). Más adelante, se realiza un tratamiento categórico de esta variable, agrupándose los datos en 
tres grupos: hasta 25 años, entre 25 y 50 años y más de 50 años.

- Estado civil: variable no numérica nominal, cuyas categorías son soltera, casada, separada, divorciada, pareja de hecho, viuda, y otro. La mayoría de las mujeres observadas, 1550 (79.4\%), están solteras. Un porcentaje ínfimo, del $0.8 \%$, corresponde a mujeres casadas y viudas. Finalmente, los porcentajes de mujeres separadas, divorciadas y en parejas de hecho son, respectivamente, del $7.6,6.1$ y $3.5 \%$.

- Hijos: variable numérica, que recoge el número hijos que son acogidos, junto a la mujer, en el recurso. Sus valores discretos oscilan entre cero y seis. Setecientas sesenta mujeres (38.9\%), el mayor número, han sido ingresadas en la casa de acogida junto con un hijo (moda de la distribución). En segundo lugar, se encuentran las mujeres con dos hijos acogidos (25\%), seguidas de aquellas que no tienen hijos acogidos (22.3\%). Los números medio y mediano de hijos acogidos con la mujer son 1.34 y uno, respectivamente.

- Ingresos: variable no numérica nominal dicotómica, con dos únicas categorías: sí o no, dependiendo de que la mujer tenga ingresos o no a su salida del recurso. En general, los ingresos proceden de la renta activa de reinserción (RAI), contemplada en el artículo 27 de la Ley Integral. Estas ayudas, que se modulan en relación a la edad y responsabilidades familiares de la víctima, tienen como objetivo fundamental facilitarle a esta unos recursos mínimos de subsistencia que le permitan independizarse del agresor. De las 1888 mujeres sobre las que se dispone de información para esta variable, 1182 (62.6\%) sí disponen de ingresos económicos a la salida del recurso.

- Tiempos de estancia: variable numérica que recoge el tiempo (en días) de la duración de la estancia de la mujer 
en el recurso. La duración media de la estancia es de 90.42 días y la duración mediana de sesenta y seis días. El valor de la media es considerablemente superior al de la mediana debido al desplazamiento que los valores especialmente elevados de la distribución ocasionan sobre la primera. De hecho, si bien la máxima observación de la que se dispone es de cuatrocientos treinta y cuatro días, el valor de ciento cincuenta y cinco días acumula el $80 \%$ de los datos. Posteriormente, consideraremos los valores que toma esta variable agrupados en tres categorías: estancia corta, estancia intermedia y estancia larga, cada una de las cuales contiene la tercera parte de los datos.

- Última residencia: variable no numérica nominal que indica la procedencia de la mujer antes de ingresar en el recurso. Se contemplan como categorías para esta variable Almería, Cádiz, Córdoba, Huelva, Jaén, Málaga, Sevilla, y otra. Un $95 \%$ de las mujeres acogidas procede de alguna provincia andaluza y sólo un 5\% proviene de algún otro lugar español o extranjero. El porcentaje mayor, un $25.2 \%$, está asociado a la provincia de Sevilla. Este porcentaje duplica al asociado a la segunda provincia andaluza de procedencia, Málaga, que es del 12.5\%.

- País origen: variable no numérica nominal. Se han contabilizado más de cincuenta países de origen diferentes de las mujeres analizadas, incluido España. De las 1953 mujeres acogidas, el porcentaje considerablemente superior corresponde a España (68.5\%), siendo inmigrantes el $31.5 \%$ restante. El mayor porcentaje de mujeres inmigrantes acogidas, el 9.3\%, es de origen marroquí, seguido inmediatamente del porcentaje de mujeres rumanas $(5.8 \%)$.

- Domicilio salida: variable no numérica nominal que recoge información sobre el domicilio de la mujer una vez que abandona el recurso, considerándose las siguien- 
tes categorías: piso tutelado, domicilio independiente, domicilio familiar, domicilio conyugal con el agresor, domicilio conyugal sin el agresor, domicilio con amigos, otra casa de acogida y otro recurso. La mayoría de las mujeres $(25.9 \%)$ se traslada a un domicilio familiar a la salida de la casa de acogida. El domicilio independiente y el piso tutelado son las categorías que le siguen inmediatamente en orden de frecuencia, con unos porcentajes asociados del $22.4 \%$ y $14.3 \%$, respectivamente. El $7.4 \%$ de las mujeres analizadas regresa, tras la casa de acogida, al domicilio conyugal con el agresor. Más adelante, se considerarán las modalidades de esta variable agregadas de la siguiente forma: domicilio conyugal con el agresor y otras situaciones.

\subsection{Análisis estadístico avanzado}

En esta sección, se lleva a cabo la aplicación de diversas técnicas estadísticas con el objetivo de diferenciar el perfil socioeconómico de las mujeres que deciden volver al domicilio familiar con el agresor del de aquellas que optan por cualquier otro domicilio a la salida del recurso, ambas después de realizar una estancia en una casa de acogida andaluza y someterse al programa de recuperación integral de la Junta de Andalucía.

\subsection{Contraste de independencia en tablas de doble entrada}

Esta sección profundiza sobre la variable domicilio salida, cuyas categorías anteriormente descritas se juntan en dos únicas: domicilio conyugal con el agresor y otras situaciones, puesto que interesa discriminar o diferenciar estadísticamente de las demás la categoría que representa el regreso con el agresor.

Concretamente, se pretende determinar si aspectos tales como la disposición o no de ingresos económicos, la última residencia de procedencia o el país de origen condicionan 
que las mujeres tengan más tendencia a regresar o no con el agresor, o si, por el contrario, no existe asociación entre las variables. Para ello, se lleva a cabo el contraste chi-cuadrado de independencia en las tablas de contingencia o doble entrada que se obtienen al cruzar cada par de variables. Valores suficientemente pequeños de la probabilidad límite del contraste ponen de manifiesto la existencia de evidencias estadísticas para rechazar la hipótesis de independencia.

En primer lugar, la Figura 1 y la Tabla 1 ponen de manifiesto que existe una clara relación (la probabilidad límite del contraste chi-cuadrado es 0.000) entre el hecho de que la mujeres tengan o no ingresos a la salida del recurso y el hecho de que regresen al domicilio con el agresor: entre las mujeres que vuelven al domicilio conyugal con el agresor, más de un $60 \%$ carece de recursos económicos; entre las mujeres que optan por cualquier otro domicilio a la salida, más del $60 \%$ cuenta con ingresos económicos.

Figura 1. Diagrama de barras para ingresos

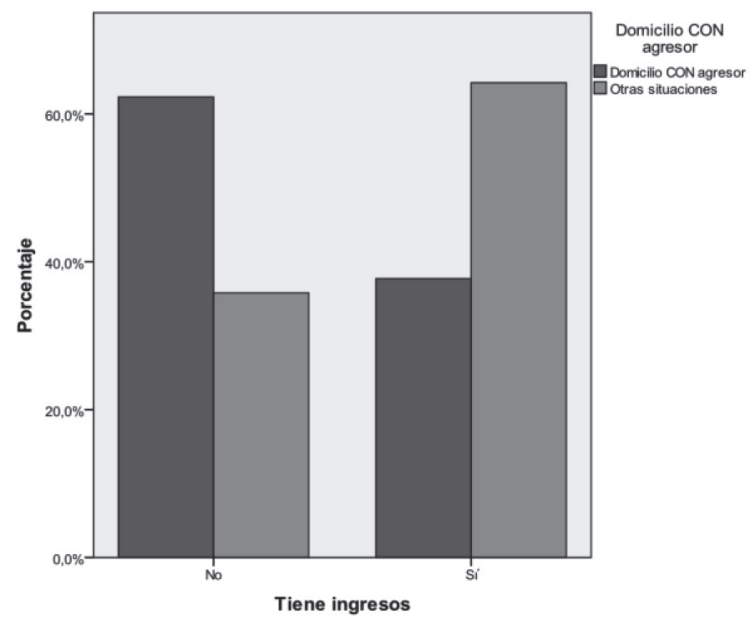

Fuente: elaboración propia. 
Tabla 1. Tabla de contingencia para ingresos (agrupando por domicilio con agresor)

\begin{tabular}{|c|c|c|c|c|}
\hline & & \multicolumn{2}{|c|}{ Domicilio CON agresor } & \multirow[b]{2}{*}{ Total } \\
\hline & & $\begin{array}{c}\text { Domicilio } \\
\text { CON agresor }\end{array}$ & $\begin{array}{c}\text { Otras } \\
\text { situaciones }\end{array}$ & \\
\hline \multirow[t]{2}{*}{ Tiene ingresos } & No & 71 & 635 & 706 \\
\hline & Sí & 43 & 1139 & 1182 \\
\hline \multicolumn{2}{|l|}{ Total } & 114 & 1774 & 1888 \\
\hline
\end{tabular}

Fuente: elaboración propia.

La Figura 2 y la Tabla 2 indican la existencia de asociación (probabilidad límite del contraste: 0.014 ) entre la provincia andaluza de última residencia de las mujeres y el posible regreso con el agresor. De las mujeres analizadas, un total de 1742 posee alguna provincia andaluza por última residencia. De ellas, ciento siete han regresado al domicilio conyugal con el agresor y las demás (1 635) se han ido a cualquier otro domicilio.

Observando la distribución por provincias de la categoría que representa la vuelta con el agresor, se observa que el mayor porcentaje corresponde a Sevilla, seguida de Huelva y Almería. Las provincias de Granada y Jaén son las que muestran un menor número de mujeres que regresan con el agresor con respecto al total de mujeres ingresadas: en Granada, de doscientas doce mujeres ingresadas en el recurso, sólo cinco vuelven con el agresor; en Jaén, de ciento veintinueve mujeres ingresadas, sólo regresan tres con el agresor. 
Figura 2. Diagrama de barras para residencia

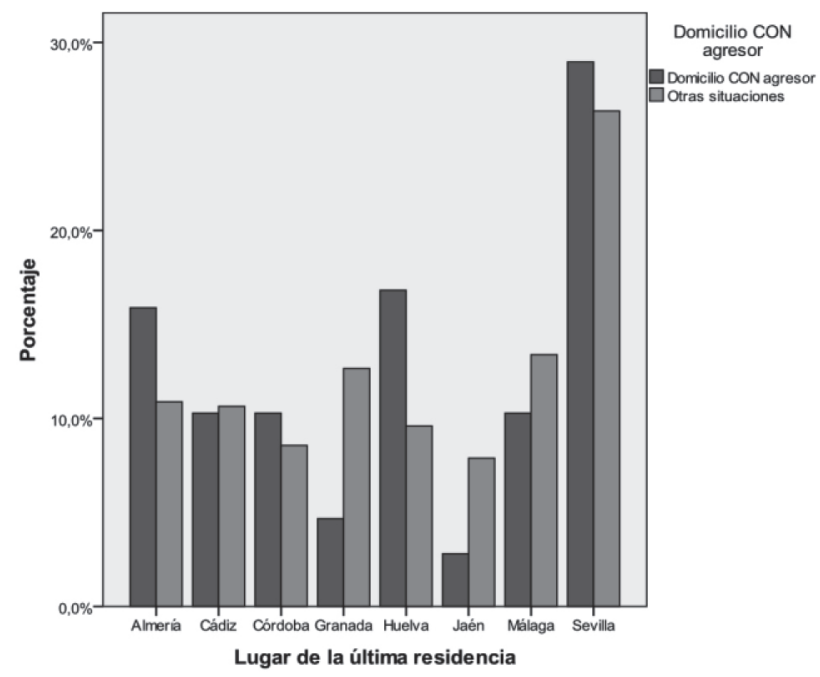

Fuente: elaboración propia.

Tabla 2. Tabla de contingencia para residencia (agrupando por domicilio con agresor)

\begin{tabular}{|c|c|c|c|c|}
\hline & & \multicolumn{2}{|c|}{ Domicilio CON agresor } & \multirow[b]{2}{*}{ Total } \\
\hline & & $\begin{array}{c}\text { Domicilio } \\
\text { CON agresor }\end{array}$ & $\begin{array}{c}\text { Otras } \\
\text { situaciones }\end{array}$ & \\
\hline \multirow{8}{*}{$\begin{array}{l}\text { Lugar de la última } \\
\text { residencia }\end{array}$} & Almeria & 17 & 178 & 195 \\
\hline & Cádiz & 11 & 174 & 185 \\
\hline & Córdoba & 11 & 140 & 151 \\
\hline & Granada & 5 & 207 & 212 \\
\hline & Huelva & 18 & 157 & 175 \\
\hline & Jaén & 3 & 129 & 132 \\
\hline & Málaga & 11 & 219 & 230 \\
\hline & Sevilla & 31 & 431 & 462 \\
\hline Total & & 107 & 1635 & 1742 \\
\hline
\end{tabular}

Fuente: elaboración propia. 
La Figura 3 y la Tabla 3 estudian el grado de relación entre la variable que representa el regreso con el agresor y los dos países de origen mayoritarios: España y Marruecos. También en este caso, se pone de manifiesto la asociación (probabilidad límite del contraste: 0.056) entre ambas variables. Así, entre las mujeres españolas ingresadas en los recursos, sólo el 5.7\% vuelve al domicilio conyugal con el agresor. Este porcentaje se eleva al 9.3\% entre las mujeres marroquíes.

Figura 3. Diagrama de barras para país

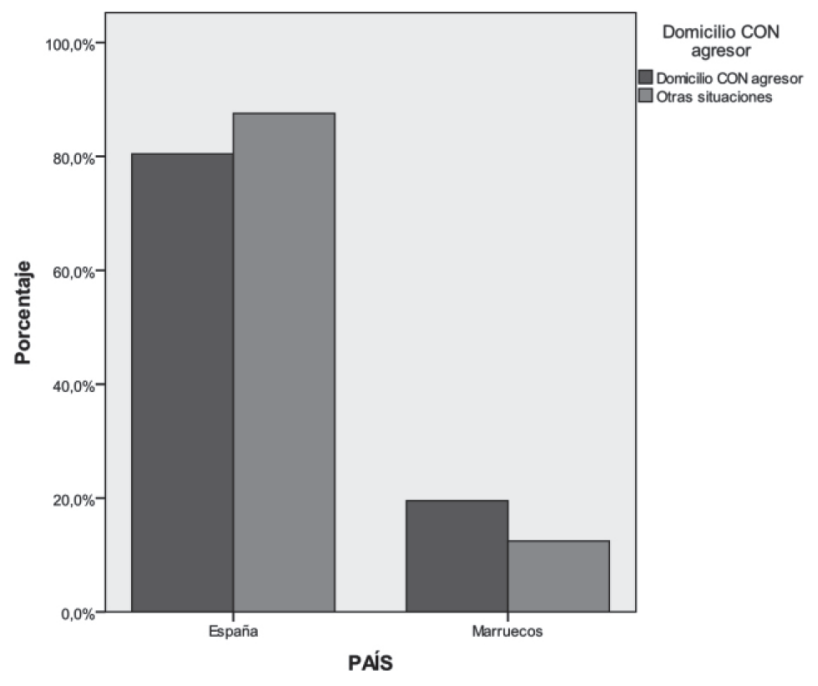

Fuente: elaboración propia. 
Tabla 3. Tabla de contingencia para país (agrupando por domicilio con agresor)

\begin{tabular}{|lll|r|r|r|}
\hline \multirow{2}{*}{} & & \multicolumn{2}{|c|}{ Domicilio CON agresor } & \\
\cline { 3 - 5 } & & $\begin{array}{c}\text { Domicilio } \\
\text { CON agresor }\end{array}$ & $\begin{array}{c}\text { Otras } \\
\text { situaciones }\end{array}$ & Total \\
\hline PAís & España & Recuento & 70 & 1161 & 1231 \\
& & $5,7 \%$ & $94,3 \%$ & $100,0 \%$ \\
\cline { 2 - 5 } & Marruecos dentro de PAís & Recuento & 17 & 165 & 182 \\
& & $\%$ dentro de PAís & $9,3 \%$ & $90,7 \%$ & $100,0 \%$ \\
\hline Total & Recuento & 87 & 1326 & 1413 \\
& & $\%$ dentro de PAís & $6,2 \%$ & $93,8 \%$ & $100,0 \%$ \\
\hline
\end{tabular}

Fuente: elaboración propia.

\section{Análisis de correspondencias}

A continuación, el objetivo es profundizar sobre la estructura de interrelaciones que existe entre las diversas modalidades de la variable domicilio salida y las variables país origen, grupos edad, estado civil y duración estancia, mediante un análisis de correspondencias. La aplicación de la técnica conlleva la construcción de un diagrama cartesiano, en dos dimensiones ortogonales, donde se representan conjuntamente las distintas modalidades para las variables analizadas. El grado de relación entre dos categorías es tanto mayor cuanto mayor es su proximidad en dicha representación cartesiana. Las modalidades con alta puntuación, en valor absoluto, en un eje de coordenadas permiten discriminar más entre categorías $\mathrm{y}$, por tanto, dar interpretación a dicho eje.

La Figura 4 muestra el diagrama cartesiano en el que se representan las distintas modalidades para la variable domicilio salida y las categorías mayoritarias para la variable país origen: España, Marruecos y Rumania. El comportamiento de las mujeres es claramente diferente en función del país de procedencia. Observando las puntuaciones en los correspondientes ejes, y pese a que el porcentaje de varia- 
bilidad de los datos explicado por cada uno de ellos no es el mismo, se deduce que la dimensión 1 (eje horizontal) discrimina entre Marruecos y España-Rumania y la dimensión 2 (eje vertical) diferencia Rumania de España-Marruecos. Con respecto a las modalidades de la variable domicilio salida, la categoría domicilio con amigos es claramente discriminada de las demás por la dimensión 2 (eje vertical).

Finalmente, observando la posible asociación entre categorías de las dos variables, se observa que:

- Las mujeres rumanas son las que tienen un comportamiento especialmente diferenciado de las demás, puesto que, en su mayoría, se trasladan a un domicilio con amigos.

- Sólo las mujeres españolas suelen trasladarse a un domicilio familiar a la salida del recurso. Sin embargo, el mayor porcentaje de ellas se traslada a otro recurso o al domicilio conyugal sin el agresor.

- La categoría que representa el regreso al domicilio familiar con el agresor no está especialmente asociada con ninguna nacionalidad, aunque es la categoría que representa a las mujeres marroquíes la más cercana a este comportamiento y la categoría que representa a las mujeres rumanas la más alejada de él.

La Figura 5 representa las distintas modalidades para la variable domicilio salida y las categorías definidas para la variable edad: hasta 25 años, entre 25 y 50 años, y más de 50 años. Aunque la edad es por naturaleza una variable numérica, se realiza un tratamiento categórico de la misma en los tres grupos considerados (que dividen a la población en tres partes de manera aproximada). De esta forma, puede establecerse la estructura de relaciones de estas categorías con las modalidades de la variable domicilio salida mediante un análisis de correspondencias. 
Figura 4. Diagrama cartesiano para las variables domicilio salida $y$ país origen

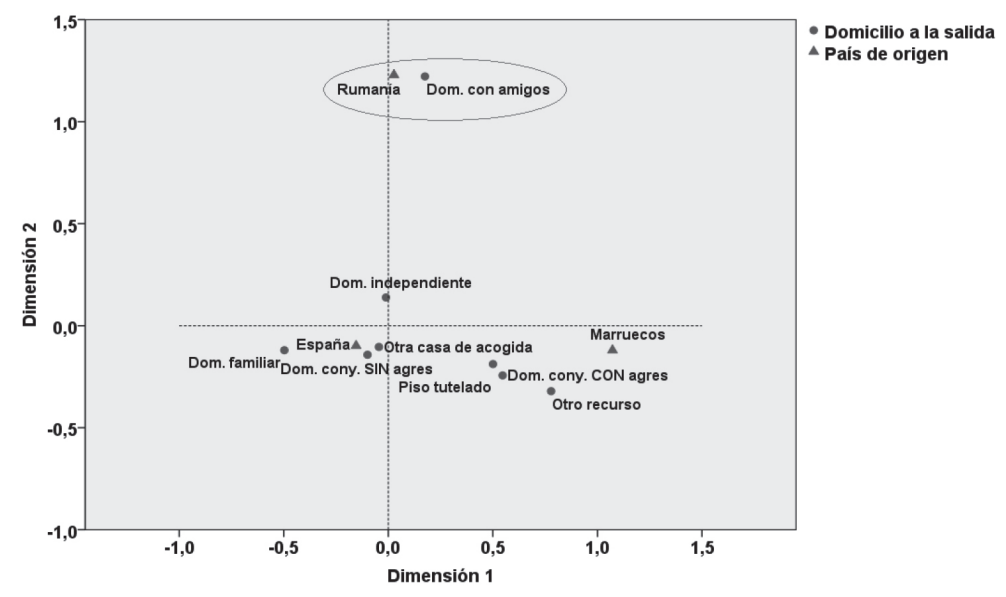

Fuente: elaboración propia.

De la observación de la Figura 5, se deduce que la dimensión 1 (eje horizontal) contrapone la salida a otro recurso con todas las demás modalidades para esta variable. La interpretación de la dimensión 2 (eje vertical), sin embargo, está relacionada con la edad de las mujeres, dado que contrapone claramente las mujeres de menos de 25 años con las de más de 50. Con respecto a la estructura de correspondencias, se deduce que:

- Las mujeres de más de 50 años son las que muestran un comportamiento más diferenciado de las demás y tienen tendencia a trasladarse a otro recurso.

- Las mujeres de edad intermedia, entre 25 y 50 años, son las que más tendencia tienen a regresar al domicilio familiar con el agresor, aunque también es habitual que pasen a otra casa de acogida, a un piso tutelado o a un domicilio independiente. 
- Las mujeres de hasta 25 años, en muchos casos, regresan a un domicilio familiar.

Figura 5. Diagrama cartesiano para las variables domicilio salida $y$ grupos de edad

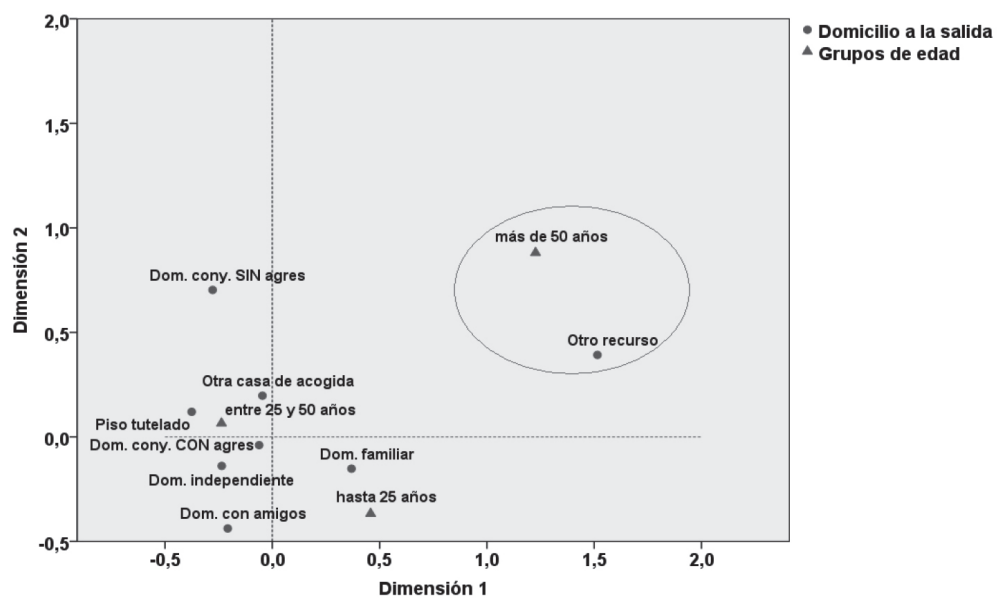

Fuente: elaboración propia.

La Figura 6 representa la estructura de correspondencias entre las categorías de las variables domicilio salida y estado civil. El gráfico pone de manifiesto que el comportamiento de las mujeres casadas es claramente diferente del de las mujeres que tienen otro estado civil. Además, las mujeres casadas no muestran ningún comportamiento respecto al domicilio de salida por carecer de asociación con alguna de las modalidades de esta variable. Por otra parte, las mujeres solteras son las que muestran una mayor tendencia a regresar al domicilio conyugal con el agresor. 
Figura 6. Diagrama cartesiano para las variables domicilio salida $y$ estado civil

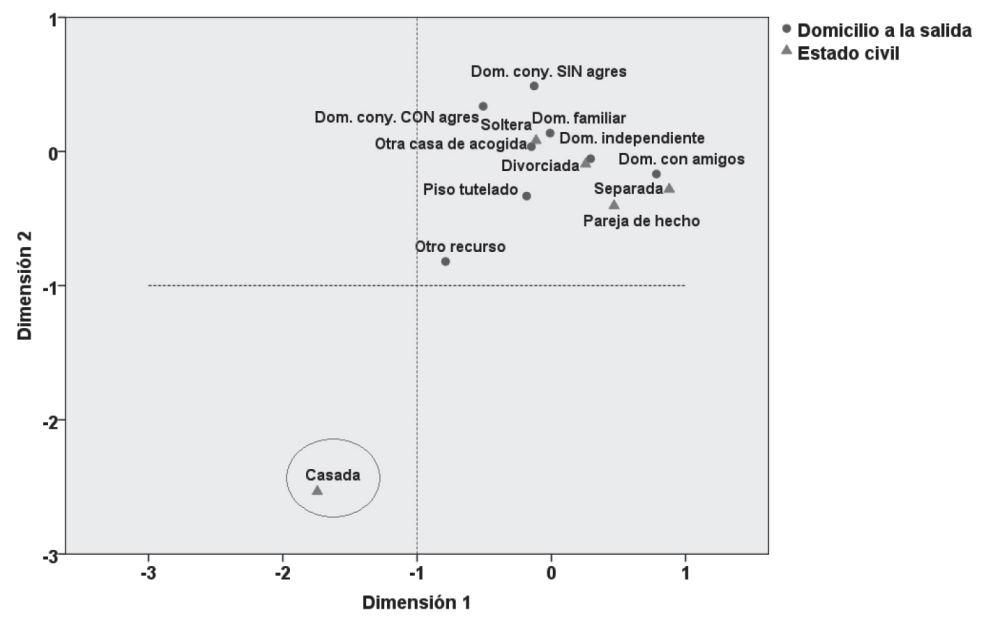

Fuente: elaboración propia.

Finalmente, la Figura 7 representa las modalidades para las variables domicilio salida y duración estancia. De esta última variable, se realiza un tratamiento categórico, considerándose las modalidades de duración de la estancia corta, intermedia y larga. La dimensión 1 (eje horizontal) representa la duración de la estancia, contraponiendo las categorías corta y larga. De la observación del gráfico, se deduce que las mujeres con una estancia larga son las que tienen mayor tendencia a trasladarse a un piso tutelado, mientras que las mujeres con estancia corta se trasladan a otra casa de acogida o al domicilio conyugal con el agresor. 
Figura 7. Diagrama cartesiano para las variables domicilio salida $y$ duración estancia

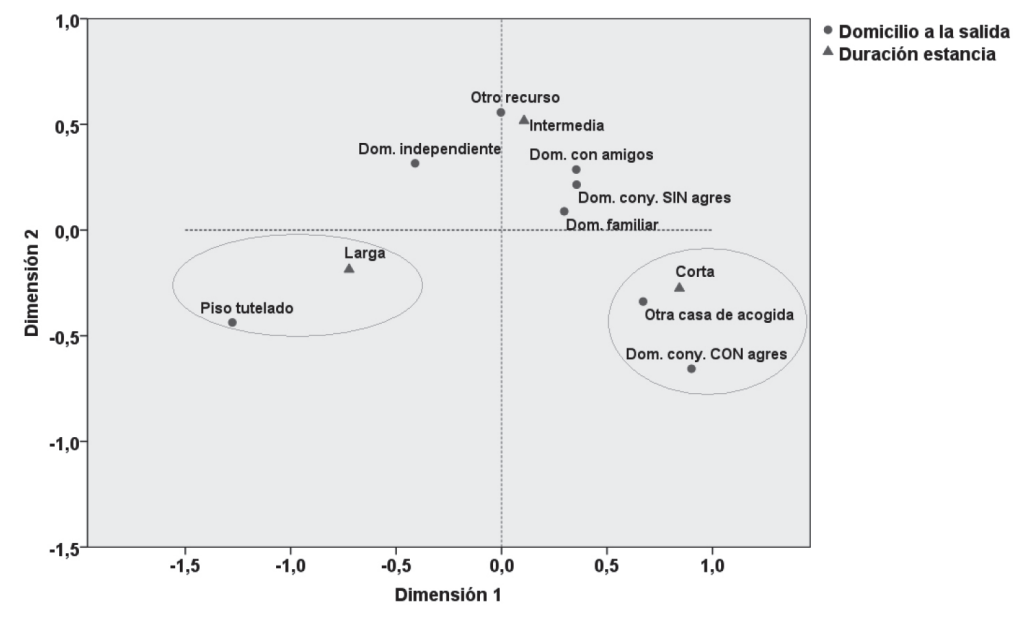

Fuente: elaboración propia.

\section{Contraste de Mann-Whitney}

En este caso, el objetivo es determinar si existen diferencias significativas en la distribución de las variables numéricas hijos, edad y tiempo estancia entre las mujeres que regresan al domicilio conyugal con el agresor y las que regresan al domicilio sin el agresor.

Antes de la aplicación de un procedimiento analítico, se detallan las intuiciones que pueden obtenerse a partir de los procedimientos gráficos. Las Figuras 8 a 10 representan las distribuciones condicionadas de las tres variables antes dichas en los grupos de mujeres que regresan al domicilio conyugal con y sin el agresor. En particular, la Figura 8 representa diagramas de barras para la variable hijos para ambos grupos de casos. De la comparación de los gráficos, se intuyen diferencias entre las dos distribuciones: las mujeres 
sin hijos o con sólo un hijo a su cargo tiene más tendencia a regresar con el agresor que las mujeres con dos o tres hijos.

Figura 8. Diagrama de barras de variable hijos para regreso a domicilio conyugal con y sin agresor

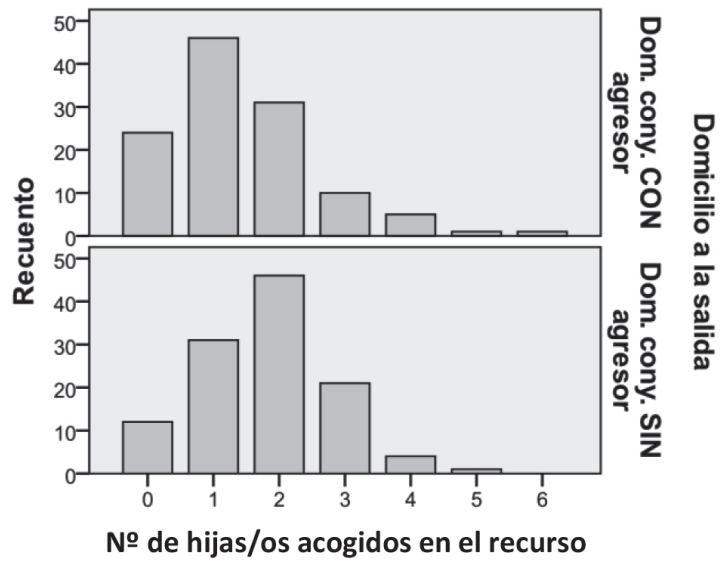

Fuente: elaboración propia.

La Figura 9 representa histogramas para la variable edad, considerando los grupos de casos que representan el regreso al domicilio conyugal con y sin el agresor. También se aprecian diferencias entre ambas distribuciones. Entre las mujeres que regresan al domicilio con el agresor, el grupo más numeroso tiene entre 20 y 40 años, presentando frecuencias similares para los intervalos de agrupación por edad (20-25, 25-30, 30-35 y 35-40 años). Sin embargo, entre las mujeres que regresan al domicilio sin el agresor, existe un intervalo modal con una frecuencia asociada considerablemente mayor: el intervalo 30-35 años. 
Figura 9. Histograma de la variable edad para regreso a domicilio conyugal con y sin agresor

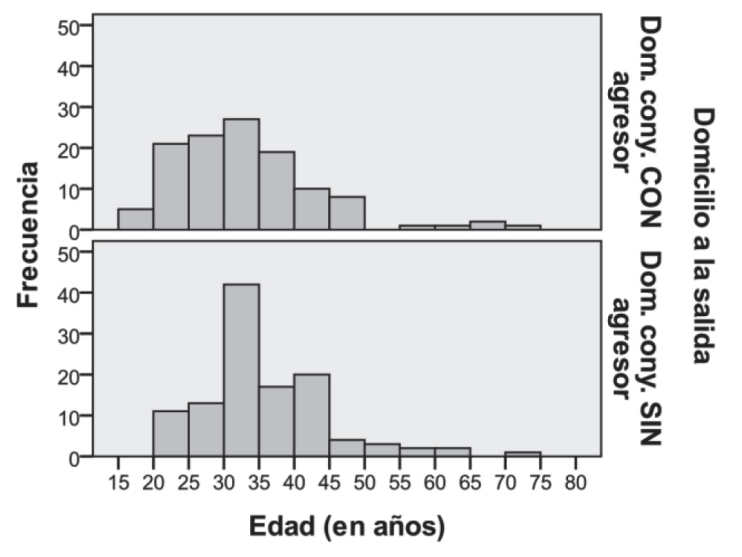

Fuente: elaboración propia.

La Figura 10 representa, para los mismos grupos de casos, histogramas para la variable tiempo estancia. Aunque para las mujeres que regresan al domicilio con el agresor la duración máxima observada de estancia en la casa de acogida es de doscientos setenta y dos días, cuarenta sobre ciento dieciocho (un $33.90 \%$ ) permanecen únicamente entre cero y doce días, y un 50\% de ellas (cincuenta y nueve mujeres sobre ciento dieciocho) permanecen hasta dieciocho días (valor correspondiente a la mediana, situada en el intervalo 12-24 días).

Sin bien la distribución de frecuencias de la variable tiempo estancia también tiene una tendencia decreciente entre las mujeres que regresan al domicilio conyugal sin el agresor, este decrecimiento se produce más paulatinamente. Así, sólo el 17.39\% de estas mujeres (veinte sobre ciento quince) permanecen menos de doce días en la casa de acogida, y la mediana en este caso asciende a cuarenta y cinco días (en el intervalo 36-48). De este modo, puede 
intuirse el efecto de recuperación integral que las mujeres consiguen a partir de la estancia en la casa de acogida.

Figura 10. Histograma de variable tiempo estancia para regreso a domicilio conyugal con y sin agresor

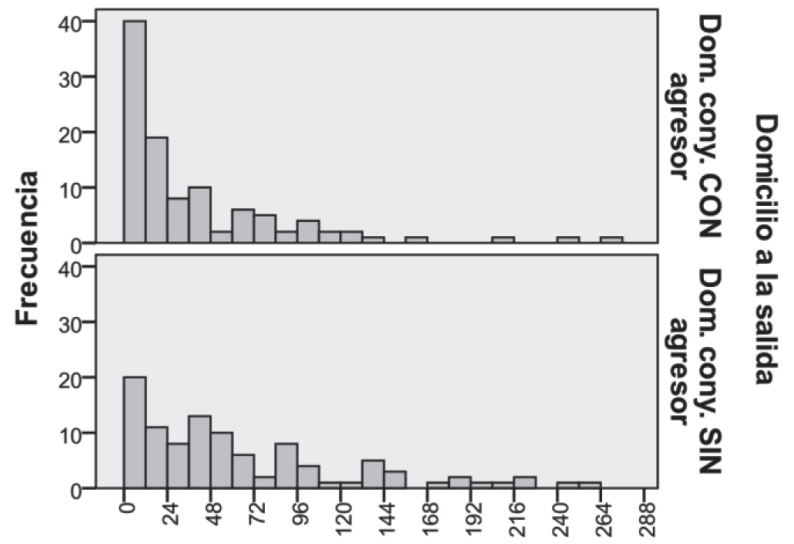

Tiempo (en días) de la estancia

Fuente: elaboración propia.

Las interpretaciones obtenidas a partir de los gráficos (Figuras 8 a 10), en este caso, las diferencias detectadas entre las distribuciones condicionadas al regreso al domicilio conyugal con o sin el agresor, han de ser confirmadas mediante un procedimiento analítico. Como la variable hijos es discreta, y ante las desviaciones respecto a la normalidad bivariante de las variables edad y tiempo estancia (ver la representación de las distribuciones condicionadas en los histogramas de las Figuras 9 y 10), se decide usar el contraste no-paramétrico de Mann-Whitney para comparar las distribuciones en los tres casos.

Este contraste plantea como hipótesis nula que la distribución de cada una de las variables (hijos, edad y tiempo estancia) condicionada al grupo de mujeres que 
regresan al domicilio conyugal con el agresor coincide con la correspondiente distribución condicionada al grupo de mujeres que regresan al domicilio conyugal sin el agresor. La hipótesis alternativa contempla la desigualdad entre las distribuciones.

La Tabla 4 recoge las probabilidades límite asociadas a cada uno de los tres contrastes (respectivamente, 0.002, 0.014 y 0.000). Al nivel de significación habitual del 5\%, se detectan diferencias estadísticamente significativas entre las distribuciones en los tres casos, por lo que se confirman las diferencias con respecto al número de hijos, la edad y el tiempo de permanencia en la casa de acogida entre las mujeres que regresan al domicilio conyugal con y sin agresor que habíamos intuido de la interpretación de los correspondientes gráficos. Esta conclusión permite establecer un perfil diferenciador entre ambos grupos de mujeres.

Tabla 4. Contraste de Mann-Whitney para variables

hijos, edad $y$ tiempo estancia

Estadisticos de contraste ${ }^{a}$

\begin{tabular}{|l|r|r|r|}
\hline & № de hijos & \multicolumn{1}{|c|}{$\begin{array}{c}\text { Edad (en } \\
\text { años) }\end{array}$} & \multicolumn{1}{c|}{$\begin{array}{c}\text { Tiempo (en } \\
\text { dias) de la } \\
\text { estancia }\end{array}$} \\
\hline U de Mann-Whitney & 5239,500 & 5517,000 & 3814,000 \\
Z & $-3,129$ & $-2,467$ & $-3,481$ \\
Sig. asintót. (bilateral) &, 002 &, 014 &, 000 \\
\hline
\end{tabular}

a. Variable de agrupación: Domicilio conyugal CON/SIN agresor

Fuente: elaboración propia.

\section{Conclusiones}

En el año 2004, la redacción de la Ley Integral consiguió, por primera vez, enfrentar el problema de la violencia de género en España de manera global, recogiendo en un 
mismo texto medidas de carácter administrativo, educativo, social y legal. Esta ley transfiere a las comunidades autónomas las competencias sobre recursos integrales de atención y acogida para las víctimas. En particular, en Andalucía se establecieron tres niveles para estos recursos, a saber: centros de emergencia, casas de acogida y pisos tutelados. Particularmente, este trabajo hizo uso de datos oficiales sobre mujeres víctimas de violencia de género ingresadas en casa de acogida en Andalucía durante los años 2004-2010, en los que, como se ha comentado, se realizaron cambios legislativos importantes en materia de violencia de género.

Inicialmente, se realizó un análisis estadístico descriptivo de dichos datos. Autores como Osborne (2009) afirman que factores como la edad o la nacionalidad son fundamentales para explicar por qué es más probable que unas mujeres tengan más posibilidades que otras de ser víctimas de violencia de género. Capella, et al. (2013) realizan un estudio descriptivo de la violencia de género para identificar los grupos de riesgo y la posible incorporación de medidas protectoras necesarias hacia los colectivos más vulnerables.

Por tanto, la descripción estadística de las variables observadas sobre las mujeres usuarias de las casas de acogida andaluzas ha constituido la primera parte de este estudio, que determina que se trata fundamentalmente de mujeres con una edad media de 31.85 años, solteras y con un único hijo ingresado junto a ellas en el recurso. Aunque se han registrado más de cincuenta países de origen, el 68.5\% de las mujeres son españolas, y más del 60\% de las mujeres consideradas tiene ingresos económicos al abandonar la casa de acogida. Con respecto a la estancia de las mujeres en la casa de acogida, puede afirmarse que las mujeres permanecen un tiempo medio de 90.42 días en el recurso, si bien la duración de la estancia más frecuente (moda) equivale a cuatrocientos treinta y cuatro días. El $80 \%$ de las mujeres permanece, como mucho, ciento cincuenta y cinco días. La 
mayoría de las mujeres comienza la estancia en la casa de acogida sin recursos, sin embargo, más del $60 \%$ de ellas recibe ayuda económica a su salida. Con respecto al lugar de residencia después de la estancia en la casa de acogida, el $25.9 \%$ de las mujeres se dirige al domicilio familiar, el $22.4 \%$ se traslada a un domicilio independiente, el $14.3 \%$ se traslada a un piso tutelado y el $7.4 \%$ por ciento regresa al domicilio conyugal con el agresor. El estudio persiguió discriminar la última categoría de las restantes.

Posteriormente, el estudio dio un paso más. Concretamente, hizo uso de técnicas estadísticas más avanzadas con la finalidad de discriminar el perfil de las mujeres que, tras someterse al programa de recuperación integral (socioeducativa, social, formativa, psicológica y jurídica), toman la decisión de regresar al domicilio conyugal con el agresor a la salida de la casa de acogida del perfil de las mujeres que optan por cualquier otro domicilio. Tal y como se indicó anteriormente, el Acuerdo de la Conferencia Sectorial de Igualdad (resolución del 09 de junio de 2015) contempla la conveniencia de que la mujer se aleje de su agresor para iniciar el proceso de recuperación.

La aplicación del contraste de independencia en tablas de doble entrada puso de manifiesto, inicialmente, que entre las mujeres que optan por el regreso con el agresor a la salida del recurso, el porcentaje mayor (60\%) corresponde a aquellas que carecen de ingresos económicos. Este dato confirmaría que las ayudas de la RAI de la Ley Integral (artículo 27) cumplen con su objetivo de facilitar a la mujer unos recursos mínimos de subsistencia que le permitan independizarse del agresor.

Con respecto al lugar de procedencia de las mujeres, las provincias de Granada y Jaén son las que muestran, en términos relativos, un menor número de mujeres que regresan al domicilio con el agresor tras la estancia. Teniendo en cuenta la nacionalidad, el porcentaje de mujeres que regre- 
san con el agresor es superior entre las mujeres marroquíes que entre las españolas. Contextualizando la información, la legislación actual en Marruecos, según Human Rights Watch, no proporciona suficiente orientación sobre la violencia de género a la policía, los fiscales o los jueces, quienes a menudo ignoran los testimonios de las víctimas y favorecen a los agresores (Human Rights Watch, 2015).

A continuación, el análisis de correspondencias profundizó en la estructura de interrelaciones que existe entre las categorías cruzadas en una tabla de contingencia. Esta técnica estadística determina que, con referencia a la nacionalidad, las mujeres de nacionalidad rumana son las que tienen un comportamiento especialmente diferenciado respecto de las mujeres de otro país de origen a la salida del recurso, ya que, principalmente, se trasladan a un domicilio con amigos. Esta conclusión coincide con la afirmación que realiza Mozota (2013) sobre el deseo de reagrupamiento familiar que se observa en la tipología rumana, que además emula a la práctica que se lleva a cabo entre los rumanos de agruparse varias familias en una casa para compartir espacio y gastos.

Respecto de la edad, las mujeres de más de 50 años tienen una clara tendencia a trasladarse a otro recurso. Según el estado civil, las mujeres solteras muestran más tendencia a regresar con el agresor, aunque las mujeres casadas son las que muestran un comportamiento más diferenciado de otras. Con respecto a la duración de la estancia, las mujeres con una mayor permanencia en el refugio tienden a trasladarse a un piso de tutelado, mientras que las usuarias que permanecen menos tiempo en la casa de acogida tienden a regresar a otra casa de acogida o al domicilio conyugal con el agresor. Este resultado confirma la efectividad del proceso de recuperación integral de las mujeres víctimas de violencia de género a mediano-largo plazo, siendo la duración media de la estancia de noventa días. 
Finalmente, la aplicación del contraste de Mann-Whitney permitió determinar que las mujeres sin hijos o con sólo un hijo tienen más tendencia a volver con el agresor que las mujeres con más hijos. Entre las mujeres que regresan al domicilio con el agresor, el grupo más numeroso tiene entre 20 y 40 años de edad; sin embargo, entre las mujeres que regresan al domicilio sin el agresor, el intervalo de 30-35 años muestra una frecuencia considerablemente mayor. Aunque la distribución de frecuencias de la variable que representa el tiempo de la estancia muestra una tendencia decreciente entre las mujeres que regresan al domicilio conyugal con el agresor y entre las que se deciden por el regreso al domicilio conyugal sin el agresor, en el segundo caso la disminución ocurre más gradualmente. Es decir, la mayoría de las mujeres víctimas de violencia de género que se deciden a regresar al domicilio con el agresor tardan muy pocos días en hacerlo, el impulso es superior recién ingresadas en la casa de acogida y, superados los primeros 10-12 días de estancia, este impulso se ve bastante reducido.

Argueelles Vázquez, R., Lorente Montalvo, P., Molero, A. C., Bibliografía y Bisol, R. (2009). "Study of cases of gender violence from analysis of judicial reports of injuries filed in a primary health center". Swiss Medical Weekly, I39(3334), I82S-182S.

Azevedo, R. G. D. (2008). "Sistema penal e violência de gênero: Análise sociojurídica da Lei II.340/06”. Sociedade e Estado, 23(I), II3-135.

Benagiano, G., Carrara, S., y Filippi, V. (2010). "Social and ethical determinants of human sexuality: 2 . Genierbased violence". European Journal of Contraception and Reproductive Health Care, I5(4), 220-23I.

Boletín Oficial de la Junta de Andalucía (1988). Ley I0/I 988, del 29 de diciembre, de Presupuesto de la Comunidad Autónoma 
Bibliografía de Andalucía para 1989. Recuperado de: http://www. juntadeandalucia.es/boja/I988// 06/d I.pdf

(2007a). Ley Orgánica 2/2007, del 19 de marzo, de Reforma del Estatuto de Autonomía para Andalucía. Recuperado de: http://www.juntadeandalucia.es/ boja/2007/56/dl.pdf

(2007b). Ley I3/2007, del I 8 de diciembre, de Medidas de Prevención y Protección Integral contra la Violencia de Género. Recuperado de: http://www.juntadeandalucia. es/boja/2007/247/2

- (2009). Orden del 06 de julio de 2009, por la que se aprueba el reglamento de régimen interno de los centros que componen el servicio integral de atención y acogida a mujeres víctimas de violencia de género y menores a su cargo que las acompañen en la Comunidad Autónoma de Andalucía. Recuperado de: http://www.juntadeandalucia. es/boja/2009/I40/34

Boletín Oficial del Estado (2004). Ley Orgánica I/2004, del 28 de diciembre, de Medidas de Protección Integral contra la Violencia de Género. Recuperado de:https://www.boe.es/ boe/dias/2004/ | 2/29/pdfs/A42 I 66-42 I 97.pdf

(2015). Resolución del 09 de junio de 2015, de la Secretaría de Estado de Servicios Sociales e Igualdad, por la que se publica el Acuerdo de la Conferencia Sectorial de Igualdad del 21 de julio de 20/4. Recuperado de: https://www. boe.es/boe/dias/2015/07/07/pdfs/BOE-A-20 I5-7620.pdf Cabrera, M., y Granero, M. J. (20I I). “Enfermería, maltrato de género y presencia de menores: redescubriendo nuestro papel”. Enfermería Global, I0(22), s/p.

Capella, M. E. T., Martin-Fumadó, C., Castro, A. M. T., et al. (20/3). "Estudio descriptivo de la violencia de género: análisis de 404 casos". Revista Española de Medicina Legal, 39(I), 7-II. 
Capella, M. L., Hill, R. P., Rapp, J. M., y Kees, J. (20I0). “The Bibliografía impact of violence against women in advertisements". Journal of Advertising, 39(4), 37-5I.

Coll-Vinent, B., Echeverría, T., Rodriguez, D., y Santiñà, M. (2007). "Family and gender violence from the point of view of health professionals". Medicina clínica, 128(8), 317-317.

et al. (2008)."Perception of gender violence by health care professionals". Gaceta Sanitaria, 22(6), 620-620.

Convención Interamericana para Prevenir, Sancionar y Erradicar la Violencia contra la Mujer (1994). Tratado multilateral de los estados partes de la convención de "Belém do Pará”. Recuperado de: http://www.oas.org/es/mesecvi/ docs/BelemDoPara-ESPANOL.pdf

Corcoy, M. (2010). "Problemática jurídico-penal y políticocriminal de la regulación de la violencia de género y doméstica". Revista de derecho (Valparaíso), 34, 305-347.

Da Mota, J. C., Godoi, A. G., y De Assis, S. G. (2007). "Correspondence analysis as a strategy for describing the profiles of women battered by their partners and assisted by a specialized unit”. Ciencia \& saúde coletiva, I2(3), 799-809.

(2008). "Correspondence analysis: A method for classifying similar patterns of violence against women". Cadernos De Saúde Publica, 24(6), I397-I 406.

Djikanovic, B., King, E. J., y Bjegovic, V. (20I3). “Gender Differences in Health Symptoms Associated with the Exposure to Physical Violence in Family: Data from the 2006 National Health Survey in Serbia". Journal of Family Violence, 28(8), 753-76I.

Echauri, J. A., Fernández-Montalvo, J., Martínez, M., y Azkarate, J. M. (20I3). "Effectiveness of a treatment programme for immigrants who committed gender-based violence against their partners". Psicothema, 25(I), 49-54. 
Bibliografía

Epstein-Ngo, Q. M., Walton, M. A., Chermack, S. T., et al. (20I3). "Analysis of daily calendar data for gender, substance use and reasons for adult partner and nonpartner violence". Alcoholism-Clinical and Experimental Research, 37, 23A-23A.

Fernández-Montalvo, J., Echauri, J. A., Martínez, M., y Azkarate, J. M. (20I I). "Gender violence and migration: differential profile of domestic violence between native and migrant men”. Behavioral Psychology, 19(2), 439-452.

Ferrer, V. A., Bosch, E., y Navarro, C. (20II). "Gender violence in higher education: Predictors factor analysis". Anales de Psicología, 27(2), 435-446.

Ferrer Pérez, V. A., y Bosch Fiol, E. (20I4).“GenderViolence as a Social Problem in Spain: Attitudes and Acceptability”. Sex Roles, 70(II-12), 506-52 I.

Hearn, J., y McKie, L. (20I0). "Gendered and Social Hierarchies in Problem Representation and Policy Processes: Domestic Violence in Finland and Scotland". Violence against Women, I6(2), I36-I58.

Human Rights Watch (2015). Informe mundial sobre Marruecos. Recuperado de: https://www.hrw.org/es/worldreport/20I5/country-chapters/268I89

Iv Conferencia Mundial de la Mujer (1995). Declaración y plataforma de acción de Beijing. Recuperado de: http://www. un.org/womenwatch/daw/beijing/pdf/BDPfA\%20S.pdf

Lemaitre, J.,y Sandvik, K. B. (20I4).“Beyond SexualViolence in Transitional Justice: Political Insecurity as a Gendered Harm”. Feminist Legal Studies, 22(3), 243-26I.

Lorente, J. A., Lorente, M., y Martínez Vilda, M. E. (2000). "Síndrome de agresión a la mujer. Síndrome del maltrato a la mujer". Revista electrónica de ciencia penal y crimonología, 2, 7. Recuperado de: http://criminet.ugr.es/ recpc/recpc_02-07.html

Martín Llaguno, M., y Navarro Beltrá, M. (20I3). “Laws on gender violence and their effect on sexism in adver- 
tising: A comparative analysis of advertisements from Bibliografía Argentina, Mexico, Spain and the United States". Revista Panamericana de Salud Pública-Panamerican Journal of Public Health, 33(4), 280-286.

Martín Martínez, M. M. (20I I)."Victims protections, gender violence and judicial cooperation in criminal matters in the EU Post-Lisbon". Revista de Derecho Comunitario Europeo, 15(39), 407-442.

Molina, D. I., Martínez Ortega,A., y Guancha,A. J. (20I3)."El control de la conducta emocional: una visión de responsabilidad penal en contra de la violencia de género". Revista Opinión Jurídica, I2(23), 67-80.

Montilla, G., Pérez-Cordón, L. G.,y Montes-Berges, B. (2010). "Towards an education aware of the gender violence problematic”, en, INTED 2010 proceedings, IATED (PP. 45354540). Valencia: INTED.

Mozota, J. G. (20I3). “El asociacionismo rumano en Aragón (I 990-20 I I)". Espacio, Tiempo y Forma. Serie V, Historia Contemporánea, (25), 30 I-326.

Mulla, S., y Hlavka, H. (20I I). "Gendered Violence and the Ethics of Social Science Research". Violence against Women, I 7(I2), I509-I520.

Muslera-Canclini, E., Natal, C., García,V.,y Fernández-Muñoz, P. (2009). "Description of registration of episodes of gender violence in medical records in the Principality of Asturias, Spain”. Gaceta Sanitaria, 23(6), 558-56I.

Osborne, R. (2009). Apuntes sobre violencia de género. Barcelona: Bellaterra.

Puigvert, L. (20I4). "Preventive Socialization of Gender Violence: Moving Forward Using the Communicative Methodology of Research". Qualitative Inquiry, 20(7), 839-843.

Rangel da Silva, L., Domingues Bernardes Silva, M., Mota Xavier de Meneses, T., et al. (20I I). “El fenómeno de la 
Bibliografía violencia de género en la mujer a partir de la producción científica de enfermería". Enfermería Global, I0(22), s/p. Rodríguez Menés, J., Puig, D., y Sobrino, C. (2014)."Polyand Distinct Victimization in Histories of Violence Against Women”. Journal of Family Violence, 29(8), 849-858.

Ruan, W. J., Dawson, D. A., Chou, P., y Grant, B. F. (2010). "Alcohol, Gender and Violence-related Injury: Data from the WHO Collaborative ED Study In China". Alcoholism: Clinical \& Experimental Research, 34(6), 55A-55A. Segura, S. (20I2). "Razonamiento contrafáctico, responsabilidad y culpa de la violencia contra las mujeres en la pareja: Educación y medios de comunicación como factores preventivos". Escritos de Psicología (Internet), 5(3), 43-5I. Recuperado de: http://scielo.isciii.es/scielo. php?script=sci_arttext\&pid=SI 989-380920 I 2000300006

Vives-Cases, C., Álvarez-Dardet, C., Carrasco-Portiño, M.,y Torrubiano-Domínguez,J.(2007)."The impact of gender inequality on intimate partner violence in Spain". Gaceta Sanitaria, 2 I (3), 242-246.

Yoon, H.J.,y Kim,Y. (20 I4).“The Moderating Role of Gender Identity in Responses to Comedic Violence Advertising". Journal of Advertising, 43(4), 382-396.

Zayas, B. (2006). “Educating for citizenship.The school prevention against gender violence”. Revista Española de Pedagogía, 64(234), 387-388.

Zuckerhut, P. (20I0). "From the discussion on violence in anthropology and social sciences to a feminist analysis of gendered violence". ZeitschriftFurEthnologie, 135(2), 275-304. 